\title{
Review I Flow: The Rhythmic Voice in Rap Music
}

Mitchell Ohriner

New York: Oxford University Press, 2019

ISBN: 9780190670412 (HB)

\section{Benjamin Burkhart \\ University of Music and Performing Arts Graz benjamin.burkhart@kug.ac.at}

Flow is an important criterion for evaluation in rap music. Whether MCs have the skills to flow or not is frequently discussed by musicians, fans, and critics. But what exactly is flow and how can it be analyzed? In his book Flow: The Rhythmic Voice in Rap Music, Mitchell Ohriner, who serves as an assistant professor of music theory at the Lamont School of Music in Denver, argues that "flow exists in a rhythmic space between music and speech" (xxiii). That is to say, the term refers to one of the many nuances that are highly relevant for the sound of popular music, but is difficult to grasp for music analysists. With his study, Ohriner aims at providing new perspectives for the detailed computer-based analysis of flow and wants "to bring the full force of the tools of computational music analysis (CMA) to bear on questions on flow" (xxiii). The following questions are at the core of the study:

What are the most typical ways of flowing? What is an accent in flow, and how is it patterned? Can rap flows have a structure that transcends the individual verse? In what ways can an emcee interact with the surrounding instrumental beat? What is the meaning of the rhythm of speech in the context of rap music? (xxiv)

The book is divided in two main parts, consisting of eight chapters. In the first chapter, Ohriner elaborates his understanding of the term flow. Chapter two is about the "defining and building of a corpus of rap verses" (xxiv), which then forms the basis for the computer-based comparative analysis of a large number of rap verses. In the following three chapters, the analytical toolkit is developed with the parameters accent, rhythm, and vocal groove being described as the categories for 
analyzing flow in rap music. The book's second part contains three case studies on the individual flows of Eminem, Black Thought and Talib Kweli.

The corpus of rap verses was compiled based on six lists of "the best emcees'" (38) from the magazines The Source Magazine, Complex Magazine, B.E.T., XXL, and the website metacritic.com. Additionally, a sample of 75 tracks by the most popular MCs (according to the lists) was defined, and complemented by several other tracks for the case studies. The verses of these tracks were transcribed manually by the author in order to file them in a machine-readable format. This enabled Ohriner to compare the verses in detail with the aid of computer-based analysis tools, and to define several recurring features. The most relevant analytical category is what the author calls vocal groove: "My model of vocal groove in rap flows [...] primarily addresses the durations between accented syllables (the 'inter-accent intervals' or IAIs of flow) and further stipulates that rap flows are usually a combination of IAls of 2 or 3 units in length" (136). Ohriner continuously contextualizes his descriptions of flow models with easy to read transcriptions.

The case studies are dedicated to specific features of the individual flows of the three aforementioned MCs. In the case of Eminem, the focus is on the complexity of groove classes and the connection of this complexity with the topics of his lyrics. Ohriner argues that "many of Eminem's verses [...] are about his own development as an emcee. To support this narrative of accruing competency, Eminem often sequences grooves such that they accrue metric complexity" (142). He further notes that there could be an "association between complexity and competency" (ibid.). In the case study on Black Thought, the author focusses on the question of "how do flow and beat complement each other?" (160). In this context, the author has two main aims: "The first is to show how flows and beats might relate beyond the Beat to Lyrics process so many emcees and critics assume [...]. The second aim is to demonstrate how we can support and constrain claims for divergence or convergence between the flow and the beat" (ibid.). The third case study is dedicated to Talib Kweli. Bearing in mind that Kweli is often considered an "'offbeat' rapper" (182), Ohriner's aim is to analyze which parameters could be relevant for that perception. He therefore focuses on the "four processes creating non alignment: phase shift, swing, tempo, and deceleration" (188), concluding that these features should rather be regarded as "a site of rhythmic expression [...] and not a detrimental feature of his rapping" (204).

Ohriner's book is an inspiring contribution to the study of vocal expression (not only) in rap music. The author illustrates that the use of computational tools, based on big datasets, can be fruitful for the in-depth analysis of musical nuances. Ohriner can show that accent structures in different rap parts can be analyzed very well in this way. Above all, the approach ensures comparability between a large number of different rap styles, which is much more difficult with non-computer-aided analysis methods. However, aspects such as microtiming or timbre, which can be regarded as just as important for flow, are taken into account to a much lesser extent - there is a need for further research here. The various transcriptions and visualizations are comprehensible for readers, too, who are not trained in reading sheet music. A valuable feature is the accompanying website, created by Oxford University Press (www.oup.com/us/flow) containing "audio excerpts that correspond to each transcription in the book" (xli). Datasets and metadata are available via https://github.com/mohriner/flowBook. These additions both make the analysis comprehensible, and the research more transparent.

The study demonstrates that flow can be regarded as a complex phenomenon. Nonetheless, it is, of course, only one feature among many others (also non- 
musical) relevant for rap music. Ohriner himself is aware of the limitations of his approach (cf. xxviii ff.). But one could argue that studying musical nuances is entirely legitimate as long as it contributes to a deeper understanding of cultural phenomena like rap music. The challenge, then, is to connect approaches of music theory as presented by Ohriner to the study of the music's reception among fans and musicians. How do they evaluate flow? Which musical features do they consider relevant? And how can results as presented in this book help to understand these evaluations by connecting music analysis with the analysis of the aesthetic judgements? In this sense, music theory and musicology can contribute to a deeper understanding of popular music's aesthetics. The various toolkits for the analysis of musical nuances illustrated in Ohriner's book can form the basis for further research on the reception of these nuances. 